\title{
Enttäuschte Meeting-Euphorie
}

Meetings sind in aller Munde. Doch ein Allheilmittel für Selbstabstimmungen in Organisationen sind sie nicht: Sie sind oftmals nicht hinreichend flexibel, angemessen und praxisnah genug und können deshalb nicht allen Kooperationserfordernissen in Unternehmen gerecht werden. Mitarbeiter müssen auch andere Wege für ihre Abstimmungsbedarfe beschreiten können. Mit der Meeting-Euphorie geraten alternative Pfade allerdings leicht ins Abseits. Dass die Verdrängung anderer Kooperationsformen folgenreich ist, wird bislang vielfach übersehen.

\section{Neue Anforderungen an Selbstabstimmung: Lösungsformel Meeting}

In flexiblen und auf das Engagement der Mitarbeiter angewiesenen Arbeitsorganisationen stoßen die traditionellen Instrumente der hierarchischen Koordinierung an ihre Grenzen. Bei den sich seit geraumer Zeit durchsetzenden dezentralen Organisationsformen, wie beispielsweise Produktund Prozessorientierung, Just-in-timeProduktion, Gruppenarbeit oder Projektarbeit, wird die Koordination der betrieblichen (Teil-)Prozesse deshalb nicht mehr allein dem Management oder den Vorgesetzten überlassen (Staehle 1999; Steinmann/Schreyögg 2000). Diese Verantwortung wird inzwischen auch auf die Schultern der Mitarbeiter verteilt. Damit tritt die selbst gesteuerte Kooperation und Kommunikation durch die Beschäftigten neben die hierarchische Koordination - oder gar an ihre Stelle. Die Fähigkeit der Mitarbeiter, sich prozessbezogen selbstständig abzustimmen, wird zugleich zu einer zentralen Anforderung an die Beschäftigten in qualifizierten Tätigkeitsbereichen sowohl der Planung und Verwaltung als auch der Produktion. Aber können die Mitarbeiter die Selbstabstimmung weitgehend nach ihren Bedürfnissen gestalten oder gibt es neue Maximen der Formalisierung?

Untersuchungen zeigen, dass die Koordination bzw. Abstimmung der Mitarbeiter untereinander keineswegs so selbst gesteuert - also frei gestaltbar - stattfindet, wie angenommen werden könnte (vgl. Böhle/Bolte 2002; Krömmelbein 2004). In den Unternehmen hat sich vielmehr eine formalisierte Form, das Meeting bzw. Planungs- und Koordinationsrunden in Form von Gremiengesprächen, für die Organisa- tion der dezentralen Koordination durchgesetzt. Das Meeting als Ort der diskursiven Koordinierung (Braczyk/Schienstock 1996) gilt nunmehr als adäquate Antwort auf die neuen Anforderungen an Kooperation und Kommunikation in Unternehmen.

\section{Widersprüchliche Kooperationsanforderungen als Analysegegenstand}

\subsection{FRAGESTELLUNG}

Lassen sich mit dem Meeting wirklich die vielfältigen Erfordernisse und Bedürfnisse an Selbstabstimmung abdecken? Wenn nicht, welche Folgen gehen mit der vorfindbaren Meeting-Euphorie einher? Die einseitige Fokussierung auf das Meeting als legitimer Ort für Abstimmungen ist problematisch. Da das Meeting Kooperation formalisiert und ein planungsbezogenes Vorgehen verlangt, stößt es an strukturelle Grenzen. Eine flexiblere Form der Kooperation ist deshalb ergänzend notwendig. Diese lässt sich als informelle und erfahrungsgeleitete Kooperation beschreiben (Böhle/Bolte 2002; Porschen/Bolte 2004). Mit der vorwiegend in Meetings organisierten und dort als legitim geltenden Selbstabstimmung der Mitarbeiter bleibt dafür allerdings kaum mehr Raum und Zeit. Was folgt daraus? Obwohl Selbstabstimmung prinzipiell als explizite Arbeitsaufgabe der Beschäftigten definiert ist, sind Mitarbeiter mit widersprüchlichen Kooperationsanforderungen konfrontiert, die sich belastend auf sie auswirken können. Um solche Nebenfolgen selbst organisierter Arbeit erfassen zu können, sind neue Wege in der Belastungsforschung notwendig.

\section{2 ANALYSEKONZEPT UND METHODIK}

Einen geeigneten Ansatz, das Verhältnis zwischen dem Anspruch an Selbstorganisation und den vorhandenen Bedingungen und Ressourcen auszuleuchten, bietet das Konzept widersprüchlicher Arbeitsanforderungen in Verbindung mit dem relationalen Belastungs-Ressourcen-Modell (Moldaschl 1991 sowie 2005, S. 255f.). Belastung wird hier analysiert als Diskrepanz zwischen dem, was eine Arbeitskraft tun soll, und den konkreten Bedingungen und Ressourcen, die ihr dafür zur Verfügung stehen. Was als Ressource bewertet wird, hängt von dem jeweiligen Verwendungszweck ab. Geraten Ziele, Ressourcen und Regeln der zu bewältigenden Arbeit aus dem Gleichgewicht bzw. entstehen widersprüchliche Verhältnisse in den Handlungskontexten und haben die Beschäftigten auf deren Vermeidung oder Verminderung keinen ausreichenden Einfluss, entstehen Belastungen. Diese werden demnach weder auf isolierbare Einzelmerkmale noch auf einen Mangel subjektiver Kompetenz zurückgeführt, sondern ergeben sich aus den jeweiligen Kontextbedingungen des Arbeitshandelns (relationaler Ansatz). Sie machen sich in Mehrarbeit und Arbeitsintensivierung, aber auch in Kompensationspraktiken wie riskantem Arbeitshandeln und Überforderung bemerkbar (vgl. Böhle et al. 1993,

Stephanie Porschen, Dr., Wissenschaftliche Mitarbeiterin am Institut für Sozialwissenschaftliche Forschung e. V. (ISF) München. Arbeitsschwerpunkte: Arbeit und Subjekt, Kooperation und Kommunikation in Unternehmen, Wissensmanagement. e-mail: stephanie.porschen@isf-muenchen.de 
S. 78f.; Moldaschl 2005, S. 255f.; Bolte et al. 2008). ${ }^{1}$

Mit diesem konzeptuellen Programm lassen sich Ursachen für neue Belastungen als Folge der Meeting-Euphorie erfassen. In unserer empirischen Studie „Belastung durch Kooperation " haben wir mittels qualitativer Leitfadeninterviews analysiert, wie in Unternehmen unter gegebenen organisatorischen Rahmenbedingungen kooperiert wird und welches Verständnis von Kooperation sich dort durchgesetzt hat. ${ }^{2}$ Mit dem vorgestellten Belastungskonzept wurden dann widersprüchliche Ziele, Regeln und Ressourcen bei der Bewältigung von Abstimmungsarbeit identifiziert. Da die Belastungen nicht auf Einzelmerkmale zurückgehen, sondern in den Verhältnissen stecken, werden sie auch als ,tacit work load" bezeichnet. ${ }^{3}$

Die Untersuchung wurde in sechs Unternehmen unterschiedlicher Größenordnungen aus verschiedenen Branchen klassischer Industriezweige durchgeführt. Das Sample bezog zwei Unternehmen des Maschinen- und Anlagenbaus, zwei Unternehmen der Elektroindustrie und je ein Unternehmen aus dem Baunebengewerbe und aus dem Automobilbau ein. Drei der Betriebe sind Großunternehmen, zwei mittelständische Unternehmen und eines ist ein Kleinunternehmen, wobei die Anzahl der Beschäftigten im Unternehmenssample von 60 bis zu mehr als 100.000 reichte. Die meisten der Gesprächspartner waren mit der Entwicklung neuer Produkte beschäftigt sei es als Entwickler und Produktmanager oder aber als deren Kooperationspartner aus der Produktion. Bei diesen Tätigkeiten steht nicht die diskursive Kommunikation zur Entwicklung von Strategien etc. im Mittelpunkt, sondern die in die Praxis eingebettete Kooperation zur Abstimmung von Produktentwicklungen und zur Organisation der Produktion. Konstruktions-Knowhow und praktisches Erfahrungswissen stehen hier im Vordergrund. Kommunikation wird deshalb auch nicht unbedingt als „eigentliche" Arbeit bewertet.

\section{Meeting-Euphorie: Chancen und Grenzen}

Mit Meetings sind natürlich nicht per se Belastungen verbunden. Wenn sie richtig durchgeführt werden, sind Meetings in un- terschiedlichen Varianten für bestimmte Zwecke der gruppenbezogenen Selbstabstimmung durchaus ein wichtiges Instrument.

\subsection{CHANCE FÜR GRUPPENBEZOGENE SELBSTORGANISATION}

Meetings sind ohne Zweifel gut dazu geeignet, Informationen zeitgleich an mehrere Mitarbeiter weiterzugeben. Durch den (zumindest offiziell) weitgehenden Konsens darüber, dass in offiziellen Gremiengesprächen getroffene und dokumentierte Beschlüsse gewichtiger sind als bloße Absprachen, ist mit dem Meeting auch eine Art Berufungsinstanz für Beschlüsse und deren Einhaltung eingerichtet. Außerdem können und sollen in Meetings Entscheidungen fallen, die die mittel- und langfristigen Strategien von Unternehmen betreffen; hier ist der Ort, um Prozesse in ihren Soll-Zuständen zu entwickeln und abzubilden und dem Unternehmen eine Richtung zu geben. Meetings sind auch dazu da, Aufgaben zu steuern und zu klären, also zu koordinieren. Damit schaffen Meetings als durchaus wünschenswerter Nebeneffekt - Transparenz: In ihnen erfahren die Beschäftigten, woran die Kolleginnen und Kollegen gerade arbeiten und für welche Aufgaben sie zuständig sind. Dafür kommen auch ganz unterschiedliche Meetingformen zum Einsatz, wie ein Streifzug durch die „Meeting-Landschaft“ zeigt: Sie reichen von Besprechungsrunden in einem regelmäßigen Turnus mit einem - zumeist - festgelegten Teilnehmerkreis (,jeden Montagmorgen um 9 Uhr") bis zu situations- und anlassbezogenen Meetings, die kurzfristig durch diejenigen einberufen werden, die mit einem Problem konfrontiert sind. Den Inhalten nach reichen die Meetings von Strategie- und Planungs- bis hin zu Arbeitsgesprächen, der Arbeitsorganisation nach von Bereichs-, Abteilungsund Werkstatt- bis hin zu Projektgesprächen bzw. Koordinationsrunden. In den verschiedenen Arbeitskulturen entwickeln sich darüber hinaus auch unterschiedliche Handhabungen. Überhaupt ist Meeting nicht gleich Meeting. Durch verschiedene Techniken können Meetings einen unterschiedlichen Charakter erlangen. Die empirischen Untersuchungen zeigen allerdings, dass Spielregeln, wie eine entsprechende Organisation, Vorbereitung und Moderation der Meetings und darüber hinaus pünktliches Erscheinen, eine Tages- ordnung und Verantwortungsverteilung bis hin zur Nachbereitung, zwar generell empfohlen, üblicherweise aber kaum eingehalten werden (Neumer 2007; Bolte et al. 2008; Kals 2008). Darüber hinaus kommen belebende Moderationstechniken mit Metaplan-Karten und neuere (Groß-)Gruppenverfahren wie Story Telling oder moderne Spielarten für kreative Besprechungsrunden nur gelegentlich zum Einsatz.

Typisch für Meetings sind dagegen die zeitliche und örtliche Festlegung der Kooperation, ihre Abtrennung vom eigentlichen Arbeitshandeln, die Dokumentation der Gesprächsergebnisse und deren $\mathrm{Zu}$ gänglichkeit für Kollegen sowie das Management. Die Selbstabstimmung in den dafür vorgesehenen Meetings findet sowohl verordnet als auch unverordnet in festgelegten Formen statt. Sie rückt idealtypisch in die Nähe des für das Management charakteristischen Handlungstypus planungsbezogenen Handelns (vgl. Böhle/ Bolte 2002, S. 57ff.; Porschen 2002, S. 14; Marx 2003; Schreyögg 2003, S. 175ff.; Bolte/Porschen 2006, S. 21ff.).

\subsection{GRENZEN DES MEETINGS UND ALTERNATIVEN}

In Unternehmen läuft aber gerade bei der Produktentwicklung nicht alles nach Plan, vielmehr gibt es zahlreiche unplanbare $\mathrm{Ab}$ stimmungsbedarfe, die bewältigt werden

\footnotetext{
1 Dieses Konzept, das sich kritisch dem Verhältnis zwischen Selbstorganisation und dem dafür zur Verfügung stehenden Handlungsspielraum widmet, wurde bisher insbesondere für die Kehrseiten flexibilisierter Arbeitsformen (z. B. der Projektarbeit) angewandt (Latniak et al. 2005). Dort wird jedoch kein Augenmerk auf die intensivierte Abstimmungsarbeit gerichtet. In einer Untersuchung zu kommunikativem Stress wird ebenfalls auf das Konzept widersprüchlicher Arbeitsanforderungen rekurriert (Krömmelbein 2004). Allerdings stehen hier die Anforderungen an die diskursive Kommunikation und deren Auswirkungen auf die IchIdentität und Interaktionskompetenz der Arbeitnehmer im Mittelpunkt. Untersucht werden vor allem die neuen Formen kommunikativen Stresses infolge des Durchschlagens hierarchischer Strukturen auf die intersubjektiv-horizontale Ebene durch die Intensivierung und Extensivierung der Kommunikation und infolge der Zunahme der Forderungen an Selbstpräsentation und Selbstvermarktung (ebd., S. 201ff.).

2 Das Projekt wurde von der Hans-Böckler-Stiftung gefördert. Zur Methode und den Ergebnissen vgl. ausführlich Bolte et al. 2008.

3 In Anlehnung an Moldaschl, der von tacit work loads in Analogie zu tacit skills spricht (Moldaschl 2005, S. 247).
} 
müssen (Böhle et al. 2004). Hierfür werden in die Praxis eingebundene Formen des Austauschs gesucht - ein kooperativer Erfahrungstransfer (Porschen 2008a). Für komplexere Entscheidungen werden darüber hinaus vorbereitende Zwischenbesprechungen jenseits von Meetings notwendig, sonst lässt sich im Gremiengespräch schwer ein Konsens herstellen (Bolte/Neumer 2008).

Für in flexibler Produktion und dezentraler Organisation auftretende Unwägbarkeiten muss im Rahmen der alltäglichen Arbeit sowohl im unmittelbaren Arbeitsbereich wie auch bereichsübergreifend mit anderen Mitarbeitern im Unternehmen ein Austausch möglich sein. Oftmals ist es auch günstig, die Fragestellungen mit den betroffenen Kollegen direkt „,vor Ort“ an den konkreten Arbeitsgegenständen zu klären. Dieses Vorgehen entspricht einer arbeitsbezogenen informellen und erfahrungsgeleiteten Kooperation. Das Attribut „erfahrungsgeleitet" bedeutet dabei, dass informelle Kooperation nicht im Sinne einer Abgrenzung zur formellen Kooperation zu verstehen ist, beispielsweise im Sinne einer „Schattenwirtschaft", sondern als eigener Handlungsmodus: Neben der situationsbedingten Anwendung und der Vermittlung der Probleminhalte über die Arbeitsgegenstände spielt hierbei auch ein geteilter Erfahrungs- und Erlebnisraum eine Rolle. In diesem Rahmen begegnen sich die Interaktionspartner weniger als konkurrierende Funktionsträger denn als kooperierende Experten ihrer jeweiligen Arbeitsgebiete (vgl. Böhle/Bolte 2002; Porschen/Bolte 2004). Hier gewinnen auch die in Meetings häufig auftretenden Selbstdarstellungsinteressen nicht so leicht die Oberhand.
Diese Form der Kooperation ist auch bei der Klärung von diffusen Problemen von großer Bedeutung, denn Meetings eignen sich hierzu kaum. In Meetings bzw. Projektsitzungen werden vielmehr bestimmte Probleme gar nicht offengelegt (vgl. Grüter et al. 2000). Dies gilt insbesondere für Probleme, die die Beschäftigten selbst (noch) gar nicht präzise artikulieren können. Wer trägt schon gerne seine Probleme auf der offenen Bühne des Meetings aus und macht sich damit angreifbar? Wer wird ein „Bauchgefühl“ im Meeting als Argument anführen? Diffuse Probleme werden in anderen Situationen gelöst, wie dem informellen Gespräch in einem vertrauenswürdigen Kreis von Kollegen, mit denen persönlich diskutiert werden kann. In einem solchen Umfeld gehen Mitarbeiter davon aus, dass eine Frage oder Unsicherheit nicht als Schwäche ausgelegt wird, sondern als Möglichkeit, verschiedene Ansätze und Lösungen erst einmal unverbindlich und eher spielerisch abzuwägen und zu prüfen oder gemeinsam neue Wege aufzutun, die, wenn sie sich als ungeeignet erweisen, auch schnell wieder verworfen werden können.

Darüber hinaus besteht in den meisten Unternehmen der Wunsch, Entscheidungen in Meetings im Konsens zu fällen schon um die Akzeptanz und die Umsetzungschancen der getroffenen Entscheidungen zu erhöhen. Aber der Weg zu solchen übereinstimmend getroffenen Entscheidungen ist lang, und er wird umso länger, je mehr Personen daran beteiligt sind. Dementsprechend schwierig wird die Realisierung dieses Wunsches im Rahmen von Meetings. In der Regel müssen ver-

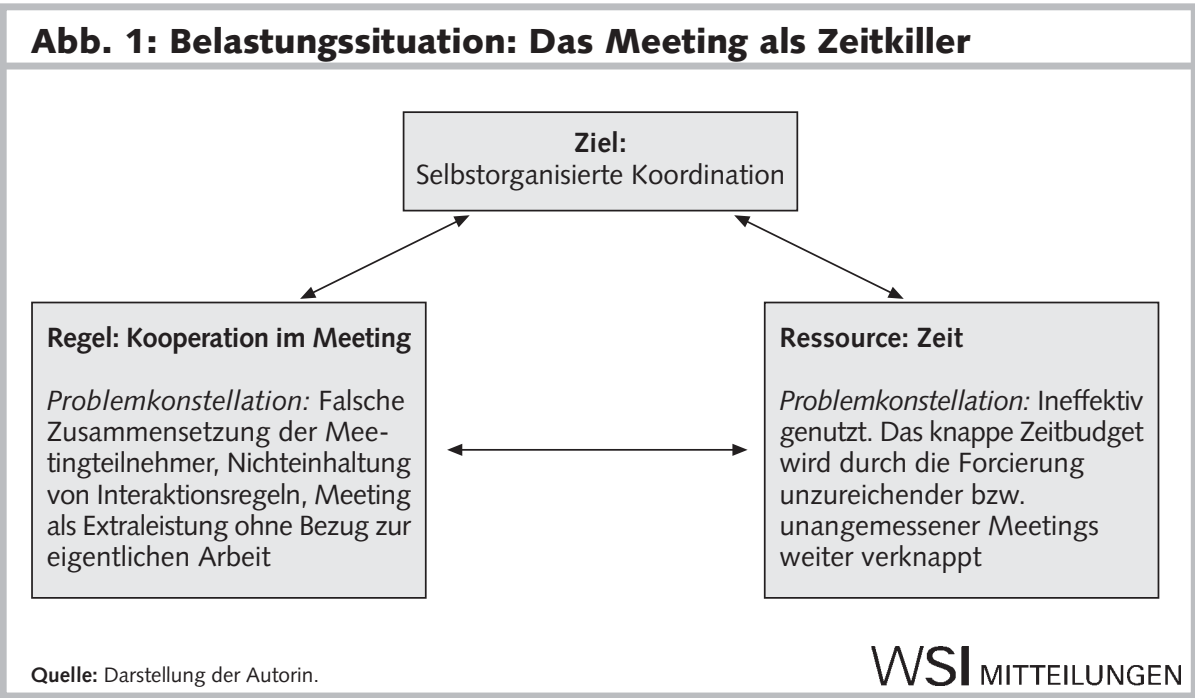

trauensvolle Face-to-Face-Gespräche im Rahmen informeller Kooperation vorausgehen.

\section{Das Meeting - nicht Lösung, sondern Teil des Problems}

Sowohl die formale Kooperation im Meeting als auch die informelle Kooperation im laufenden Arbeitsprozess ist notwendig. Wie die im Folgenden dargestellten Untersuchungsergebnisse aufzeigen, kommt es durch die häufige Einberufung von Meetings, deren oft unzureichende Organisation und aufgrund der in das Meeting hineingetragenen, dort aber kaum zu lösenden Probleme aus der Meeting-Umwelt allerdings nicht selten zu widersprüchlichen Abstimmungsbedingungen. Die MeetingEuphorie hält Mitarbeiter von der ,eigentlichen Arbeit" bzw. von einer für die Bewältigung ihrer Arbeit adäquateren Form der Kooperation ab. Mitarbeiter erleben und erleiden das Meeting deshalb oft als Zeitkiller, als Entscheidungskiller, als Dilemma der Problemgenese oder als Absicherungszwang unter Experten (Neumer 2007, S. 111ff.; Bolte et al. 2008, S. 69ff.). Im Folgenden werden die bei der Abstimmungsarbeit hinter den Geduldsproben Zeitkiller und Entscheidungskiller stehenden widersprüchlichen Verhältnisse genauer dargestellt.

\subsection{DAS MEETING ALS ZEITKILLER}

Das Spannungsverhältnis zwischen Zielen, Regeln und Ressourcen lässt sich bereits innerhalb des Meetings aufzeigen:

In den untersuchten Unternehmen gibt es - wenn auch in unterschiedlichem Ausmaß - die maßgebliche Regel, selbst organisierte Kooperation als formalisierte Kooperation in Meetings zu organisieren. Dies aber steht in einem widersprüchlichen Verhältnis zur knappen Ressource Zeit ( $A b$ bildung 1). Grund dafür ist eine Problemkonstellation, die beim formalisierten Meeting häufig auftritt: Die Meetings sind nicht immer mit dem passenden Teilnehmerkreis besetzt, Interaktionsregeln werden häufig nicht eingehalten und die Gremiengespräche weisen oft keinen Bezug zur eigentlichen Arbeit auf. Die knappe Ressource Zeit wird hierdurch ineffektiv genutzt. Wenn viele unzureichend organi- 
sierte Meetings stattfinden und wenn nicht überprüft wird, ob diese Meetings überhaupt notwendig sind, wird die Zeit für alternative Vorgehensweisen noch knapper ein Teufelskreis, der kein Einzelfall in unseren Untersuchungen war.

\subsection{DAS MEETING ALS ENTSCHEIDUNGSKILLER}

Die Beschreibung des Meetings als Entscheidungskiller resultiert aus Problemen, die in das Meeting hineingetragen werden. Wenn das Ziel, Arbeitsprozesse zu steuern und aufrechtzuerhalten, z. B. mit dem Fehlen der notwendigen Ressource Entscheidungskompetenz zusammentrifft, sind die Mitarbeiter mit dadurch verursachten widersprüchlichen Voraussetzungen für ihre Abstimmungsarbeit konfrontiert.

In dezentralen Organisationsformen ist es des Öfteren unklar, mit welcher Reichweite Teilnehmer Entscheidungen treffen dürfen. Diese Kompetenzen sind oftmals nicht definiert bzw. zugewiesen oder verteilt worden. Darüber hinaus führt eine Misstrauenskultur, die durch die Regel der Null-Fehler-Toleranz heraufbeschworen wird, zu Ängsten vor negativen Sanktionen als Konsequenz falsch getroffener Entscheidungen. Wenn falsche Entscheidungen inakzeptabel sind und diejenigen, die falsch entscheiden, dafür persönlich verantwortlich gemacht werden, wird tendenziell die Entscheidungsfähigkeit beeinträchtigt (Abbildung 2). Denn über zukünftige Entwicklungen gibt es prinzipiell kaum Gewissheiten, es können nur Annahmen getroffen werden.

Die Barrieren für Entscheidungsfindung werden erhöht durch die Vorstellung einer perfekt rationalen Entscheidung. Demnach müssten alle relevanten Informationen komplett als Faktenwissen vorliegen, um anschließend systematisch bewertet werden zu können. Im Meeting sollen dann im Sinne der diskursiven Rationalität alle Fakten abgeglichen werden. Dieses Vorgehen ist jedoch illusorisch, da im Unternehmensalltag weder alle Fakten vorliegen können noch genügend Abstimmungszeit für die Überprüfung aller möglichen Folgen vorhanden ist.

Vor diesem Hintergrund bewegen sich die Mitarbeiter - unabhängig von der Qualität der von ihnen gesammelten Fakten permanent auf unsicherem Terrain, wenn sie versuchen, die Ziele zu erreichen, die vom Unternehmen eingefordert werden.

Abb. 2: Belastungssituation: Das Meeting als Entscheidungskiller

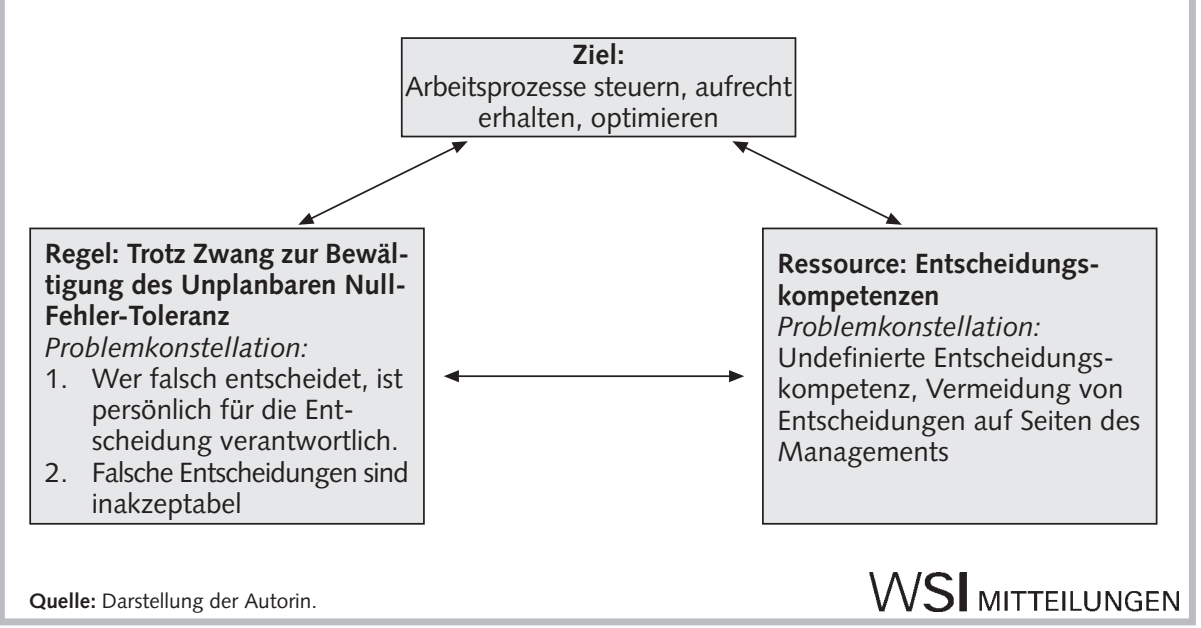

Die weitestmögliche Abschiebung von Verantwortung liegt nahe. Im Meeting kulminieren die widersprüchlichen Anforderungen, da es als Austragungsort für die geschilderten Entscheidungsdilemmata missbraucht wird. Es kann zu gegenseitigen Schuldzuweisungen kommen, die ein konstruktives Gesprächsklima trüben. Für viele Mitarbeiter besteht nicht die Möglichkeit, sich den Meetings zu entziehen, um die Widerspruchskonstellationen aufzulösen: Zum einen ist die Teilnahme an bestimmten Meetings obligatorisch. Zum anderen gehen Beschäftigte bei Nichtteilnahme immer das Risiko ein, relevante Informationen nicht zu erhalten. Für die tatsächlich angemessenen Abstimmungen und einen kooperativen Erfahrungstransfer zur Erledigung der "eigentlichen“ Aufgaben wird mitunter zeitliche und persönliche Entgrenzung sowie eine Intensivierung der Arbeit notwendig. Aus den hier exemplarisch vorgestellten Widerspruchskonstellationen erwachsen demnach neue Belastungen. Die tiefer liegenden Gründe dafür sind in dem weit verbreiteten Planungsmythos und einer in manchen Fällen feststellbaren „übertriebenen“ Angst vor prinzipiell unvermeidbaren - Risiken zu suchen.

\section{"Gesunde" Balance zwischen formeller und informeller Kooperation}

Wie ist den Nebenfolgen der Meeting-Euphorie zu entkommen, die die Kooperation in Unternehmen einseitig kanalisieren?
Wie kann die Kooperation jenseits des Meetings adäquat unterstützt werden? Dazu zeichnen sich auf der betrieblichen Organisationsebene folgende direkte Lösungswege ab.

\subsection{MEETINGS OPTIMIEREN}

Die häufig beklagte mangelnde Effizienz und Effektivität der Meetings regt zu einer kritischen Bestandsaufnahme an. Zu hinterfragen wäre zunächst, ob die im Unternehmensalltag zahlreichen Meetings alle notwendig - oder ob sie vielleicht zu zahlreich - sind. Bei den tatsächlich notwendigen Meetings lässt sich zudem beleuchten, ob sie angemessen durchgeführt werden. Zwei Beispiele aus der Praxis zeigen Umsetzungsmöglichkeiten auf: In dem einen Unternehmen wurde von dem dort neu eingerichteten unternehmenskulturellen Veränderungskreis das Thema uneffizienter und ineffektiver Meetings auf die Agenda gesetzt und ein Schulungsprogramm für Meetings entwickelt. Dieses zielte zum einen auf die Optimierung der Kommunikation, Präsentation, Moderation und Rhetorik in den Meetings ab. Vor allem gewann dieses Programm aber durch seine breite Anlage eine besondere Bedeutung: Eingeladen wurde ein Großteil der Gesamtbelegschaft mit Repräsentanten aus den verschiedenen betrieblichen Hierarchieebenen. Da sowohl Mitarbeiter als auch Führungskräfte teilnahmen, wurde hierdurch zugleich die Anerkennung der für Meetings geltenden Regeln und Methoden durch das Management gefördert. Darüber hinaus wurden die Mitarbeiter dazu angehalten, auch schnellere und direktere Wege des Austauschs zu nutzen und die Notwendigkeit 
zur Einberufung eines Meetings bewusst zu überprüfen (Bürgermeister 2008, S. 28).

Ein anderes Beispiel zeigt, wie ein Meeting von in Produktverantwortung stehenden Fertigungsmitarbeitern optimiert wurde. Dieses Abstimmungsforum war infrage gestellt worden und stand kurz vor der Auflösung. Es wurde ein kurzer Workshop einberufen, dem eine Befragung zur Funktion und Gestaltung dieses Treffens mittels eines Fragebogens voranging. Dabei stellte sich heraus, dass das Meeting als zentrales Sprachrohr der Fertigung durchaus bestimmte Zwecke erfüllte. In dem Workshop und der dadurch angestoßenen Kommunikation mit der Fertigungsleitung wurde deutlich, dass es allerdings den aktuellen Erfordernissen und Möglichkeiten angepasst werden musste. So wurde das Meeting nach dem Workshop statt in einem wöchentlichen nunmehr in einem 14-tägigen Rhythmus durchgeführt. Auch der Teilnehmerkreis ist nun flexibel erweiterbar. Wenn es thematisch weiterführend erscheint, werden Mitarbeiter aus angrenzenden Bereichen, wie beispielsweise aus dem Lager, zu diesem Abstimmungsforum der Fertigung eingeladen (Porschen 2008a, S. 42).

\subsection{ALTERNATIVE STRUKTUREN FÜR KOOPERATION ETABLIEREN}

Wenn die Grenzen der Meetings bewusst geworden sind, können darüber hinaus Gestaltungsmöglichkeiten für die arbeitsbezogene informelle Kooperation aufgegriffen und ausgebaut werden. Damit wird der flexible Wissensfluss quer über verschiedene Bereiche sichergestellt. Arbeitsorganisatorische und personalpolitische Maßnahmen, wie beispielsweise Tandemund Rotationsmodelle oder personale Netze, die sich in Unternehmen in unterschiedlichen Ausprägungen finden lassen (Bolte/Porschen 2006), schaffen dafür Gelegenheitsstrukturen.

Das Tandemmodell dient speziell zur Verbesserung der bereichsübergreifenden Kooperation an der Schnittstelle zwischen zwei Unternehmensbereichen. Das Tandem, bestehend aus Vertretern beider Bereiche, ist für die Erledigung der an der Schnittstelle anfallenden Aufgaben zuständig. Durch die Zusammenarbeit in einem gemeinsamen Erfahrungsfeld wird es den Mitgliedern möglich, die eigene Perspektive auf ein Problem um die Sichtweise anderer zu ergänzen bzw. diese gleich mitzu- denken. Die Mitglieder des Tandems sind frei in ihrer Entscheidung, wann die Kooperation erforderlich ist und wie sie jeweils realisiert wird. Auch das Rotationsmodell macht sich die Möglichkeit zunutze, vorausschauendes Handeln zu fördern. Das durch eine aktive Personalpolitik realisierbare Modell ermöglicht es Mitarbeitern, in verschiedene Bereiche des Unternehmens zu wechseln. Ziel hierbei ist, dass die Mitarbeiter verschiedene Sichtweisen und Arbeitslogiken kennenlernen. Die jeweils andere Perspektive kann so, wenn erforderlich, zukünftig besser eingebunden und nicht zuletzt rechtzeitig in Beratungsund Verhandlungssituationen eingebracht werden (Bolte 2008, S. 66ff.).

Je umfassender und komplexer nun die zu bearbeitenden Aufgabengebiete und je zahlreicher die damit befassten Organisationseinheiten sind, desto größer wird die Notwendigkeit, die potenziellen Ansprechpartner kennenzulernen. Ein besonders wirksames Modell für die Vernetzung der Teilnehmer untereinander sind Einarbeitungsprogramme beim Eintritt in die Berufstätigkeit. Die Beteiligten lernen einander von Angesicht zu Angesicht in einer Situation kennen, in der sie Zeit und Gelegenheit haben, gemeinsame Interessen und Arbeitsfelder zu eruieren; zudem unterstützen die gegenseitige Hilfe bei der Bewältigung von Aufgaben und das mit der Zugehörigkeit zu einer Gruppe verbundene Gemeinschaftsgefühl die Entstehung personaler Netze. Insofern erfüllen Fortund Weiterbildungsangebote von Unternehmen jenseits ihrer eigentlichen Zielsetzung allein schon als übergreifendes Netzwerkmodell einen wichtigen Zweck: Die geknüpften personalen Netze ermöglichen den Beschäftigten im späteren Arbeitsgeschehen eine schnelle und direkte Kontaktaufnahme zur Abstimmung und zum Austausch diverser Problemstellungen (Porschen 2008b).

Solche und ähnliche Modelle schaffen Voraussetzungen für eine tatsächlich selbst organisierbare Kooperation. Sie stellen zum einen Strukturen für die notwendige Kooperation zur Verfügung. Zum anderen ist mit ihnen genügend Offenheit für spontan auftretende Erfordernisse gegeben. Allerdings ist dafür die Akzeptanz und Unterstützung durch Führungskräfte eine wesentliche Voraussetzung. Sie müssen ein Gespür für verschiedene Möglichkeiten der Kooperation im Unternehmen entwickeln und dafür geeignete Strukturen unterstüt- zen. Beispielsweise in Form der genannten Modelle, die natürlich an die jeweiligen Anliegen in den Unternehmen angepasst sein müssen. Ausgewogene Abstimmungskulturen basieren letztlich auf einer erfahrungsbasierten Vertrauenskultur im Unternehmen, die ohne Engagement des Managements nicht zustande kommen kann.

\section{Resümee}

Meetings gelten allgemein als zeitgemäße Form der unternehmensinternen Kommunikation und Kooperation. Sie sind en vogue, sodass man zugespitzt von einer gewissen Meeting-Euphorie sprechen kann. Jedoch: Die Gestaltung vieler Meetings entspricht weder den Ansprüchen der Teilnehmer noch befördert sie unbedingt die von Meetings erwarteten Selbstabstimmungsprozesse. Auch zeigen sich Konstellationen, in denen in der Form des Meetings ritualisierte Abstimmungskulturen andere Kooperationsformen, insbesondere arbeitsorientierte informelle Kooperationen, in den Schattenbereich individueller Zusatzinvestitionen verdrängen. Für die Mitarbeiter aber heißt dies: Es kann zu subjektiv unterschiedlich empfundenen Belastungen kommen; einerseits durch unangemessen durchgeführte und überstrapazierte Meetings, andererseits durch die Zurückdrängung anderer Kooperationsformen. Solche Nebenfolgen der MeetingEuphorie legen eine besondere Facette der Entgrenzung von Arbeit offen. Dass dies bisher kaum berücksichtigt wurde, liegt in der Natur von „tacit work loads“. Beschäftigte sind nicht unbedingt in der Lage, ihre Belastungsgrenzen in flexibilisierten Arbeitsverhältnissen zu erkennen - oder sie verdrängen sie. Und erst recht ist es verpönt, sie zu artikulieren. Hier einerseits für Belastungsgrenzen zu sensibilisieren bzw. sie zu enttabuisieren, andererseits geeignete Ansätze und Methoden zur Prävention und Intervention aufzuzeigen, kristallisiert sich zunehmend als eine wichtige zukünftige Aufgabe für die Arbeitsforschung und -politik heraus. Die aufgezeigten pragmatischen Lösungswege aus der Belastungsfalle sind dazu freilich nur erste Schritte. 
Böhle, F./Moldaschl, M./Rose, H./Weishaupt S. (1993): Neue Belastungen und Risiken bei qualifizierter Produktionsarbeit, in: ISF München et al. (Hrsg.): Handbuch sozialwissenschaftliche Technikberichterstattung 1993 - Schwerpunkt: Produktionsarbeit, Berlin, S. 67-137

Böhle, F./Bolte, A. (2002): Die Entdeckung des Informellen - Der schwierige Umgang mit Kooperation im Arbeitsalltag, Frankfurt/New York Böhle, F./Bolte, A./Porschen, S. (2004): Kooperation als Belastung, in: WSI-Mitteilungen 2, S. 96-101

Bolte, A. (2008): Modelle zur Unterstützung informeller Kooperation im Arbeitsalltag, in: Clases, C./Schulze, H. (Hrsg.): Kooperation konkret!, Lengerich, S. 59-71

Bolte, A./ Neumer, J. (2008): Entscheidungsfindung in Meetings: Beschäftigte zwischen Hierarchie und Selbstorganisation, in: Arbeit, Zeitschrift für Arbeitsforschung, Arbeitsgestaltung und Arbeitspolitik, im Erscheinen

Bolte, A./Porschen, S. (2006): Die Organisation des Informellen - Modelle zur Organisation von Kooperation im Arbeitsalltag, Wiesbaden Bolte, A./Neumer, J./Porschen, S. (2008): Die alltägliche Last der Kooperation - Abstimmung als Arbeit und das Ende der Meeting-Euphorie,

Berlin

Bürgermeister, M. (2008): Veränderungskreis und Integrunt-Promotor, in: Böhle, F./Bolte, A./Bürgermeister, M. (Hrsg.): Die Integration von unten - Der Schlüssel zum Erfolg organisatorischen Wandels, Heidelberg, S. 21-34

Braczyk, H. J./Schienstock, G. (1996): Im „Lean-Express“ zu einem neuen Produktionsmodell?, in: Braczyk, H. J./Schienstock, G. (Hrsg.): Kurswechsel in der Industrie - Lean Production in Baden-Württemberg, Stuttgart/Berlin/Köln, S. 269-329

Grüter, B./Breuer H./ Wollenberg, A. (2000): Genese von Wissen in aufgabenorientierten Gruppen - Eine Fallstudie zur Wissensarbeit in der kommerziellen Software-Entwicklung, in: Witte, E. H. (Hrsg.): Leistungsverbesserungen in aufgabenorientierten Kleingruppen. Beiträge des 15. Hamburger Symposiums zur Methodologie der Sozialpsychologie vom 15. bis zum 16. Januar, Lengerich

Kals, U. (2008): Das trifft sich gut, in: Frankfurter Allgemeine Zeitung 74 vom 29./30.03., S. C1
Krömmelbein, S. (2004): Kommunikativer Stress in der Arbeitswelt Zusammenhänge von Arbeit, Interaktion und Identität, Berlin Latniak, E./Gerlmaier, A./Voss-Dahm, D./Brödner, P. (2005): Projektarbeit und Nachhaltigkeit - Intensität als Preis für mehr Autonomie?, in: Modaschl, M. (Hrsg.): Immaterielle Ressourcen, Nachhaltigkeit von Unternehmensführung und Arbeit I, München/Mehring, S. 281-314

Marx, S. (2003): Kommunikation im Arbeitsteam - Ein Fallstudie mit Ingenieurinnen und Ingenieuren, Frankfurt/New York

Moldaschl, M. (1991): Widersprüchliche Arbeitsanforderungen - Psychische Belastungen und doppelte Realität in der Produktion. Mitteilungen des SFB 333 der Universität München 3, S. 15-50

Moldaschl, M. (2005): Ressourcenorientierte Analyse von Belastung und Bewältigung in der Arbeit, in: Moldaschl, M. (Hrsg.): Immaterielle Ressourcen - Nachhaltigkeit von Unternehmensführung und Arbeit I, München/Mering, S. 243-280

Neumer, J. (2007): Und täglich ruft das Meeting ... - Eine Fallstudie über die Ambivalenzen selbstgesteuerter Abstimmung im Unternehmen, ISF München Forschungsberichte, München

Porschen, S./Bolte, A. (2004): Erfahrungsgeleitete kooperative Arbeit, in: Böhle, F./Pfeiffer, S./Sevsay-Tegethoff, N. (Hrsg.): Die Bewältigung des Unplanbaren - Fachübergreifendes erfahrungsgeleitetes Arbeiten und Lernen, Wiesbaden, S. 78-98

Porschen, S. (2008a): Agile Kooperation und Kommunikation zur prozessnahen Integration, in: Böhle, F./ Bolte, A./ Bürgermeister, M. (Hrsg.): Die Integration von unten - Der Schlüssel zum Erfolg organisatorischen Wandels, Heidelberg, S. 35-48

Porschen, S. (2008b): Austausch impliziten Erfahrungswissens - Neue Perspektiven für das Wissensmanagement, Wiesbaden

Schreyögg, G. (2003): Organisation - Grundlagen moderner Organisationsgestaltung, 4. Aufl., Wiesbaden

Staehle, W. (1999): Management - Eine volkswirtschaftliche Perspektive, München

Steinmann H./Schreyögg, G. (2000): Management. Grundlagen der Unternehmensführung, Wiesbaden 